\title{
Glucose control positively influences patient outcome: a retrospective study
}

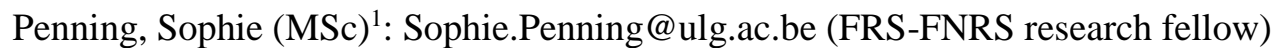

Pretty, Chris $(\mathrm{PhD})^{2}$ : chris.pretty@ canterbury.ac.nz

Preiser, Jean-Charles $(\mathrm{PhD})^{3}$ : Jean-Charles.Preiser@erasme.ulb.ac.be

Shaw, Geoffrey M. (MBChB) ${ }^{4,5}$ : geoff.shaw@cdhb.govt.nz

Chase, J. Geoffrey (PhD)²: geoff.chase@ canterbury.ac.nz

Desaive, Thomas $(\mathrm{PhD})^{1}$ : tdesaive@ulg.ac.be

\section{Affiliations :}

${ }^{1}$ GIGA-Cardiovascular Sciences, Institut de Physique, Université de Liege, Institut de Physics, Allée du 6 Août, 17 (Bât B5), B4000 Liege, Liege, Belgium.

${ }^{2}$ Department of Mechanical Engineering, Centre for Bio-Engineering, University of Canterbury, Christchurch, Private Bag 4800, 8054, New Zealand.

${ }^{3}$ Department of Intensive Care, Erasme University Hospital, 808 route de Lennik, B1070 Brussels, Belgium.

${ }^{4}$ University of Otago Christchurch, School of Medicine, Christchurch, 8054, New Zealand

${ }^{5}$ Dept of Intensive Care, Christchurch Hospital, Christchurch, 8054, New Zealand

Acknowledgements (Financial Support):

Fonds National de la Recherche Scientifique (F.R.S.-FNRS, Belgium).

\section{Corresponding authors:}

S. Penning, email: Sophie.Penning@ulg.ac.be; tel.: +32-4366-3650.

Dr. T. Desaive, email: tdesaive@ulg.ac.be; tel.: +32-4366-3733.

Prof J.G. Chase, email: geoff.chase@ canterbury.ac.nz; tel.: +64-3-364-2987 x7224. 


\begin{abstract}
Objective: The goal of this research is to demonstrate that well-regulated glycemia is beneficial to patient outcome, regardless of how it is achieved.

Methods: This analysis used data from 1701 patients from two, independent studies. Glycemic outcome were measured using cumulative Time In Band (cTIB), calculated for three glycemic bands and for threshold values of $\mathrm{t}=0.5,0.6,0.7$ and 0.8 . For each day of ICU stay, patients were classified by cTIB, threshold and hospital mortality, and odds of living (OL) and odds ratio $(\mathrm{OR})$ were calculated.

Results: OL given cTIB $\geq t$ is higher than OL given cTIB $<t$ for all values of $t$, every day, for all three glycemic bands studied. The difference between the odds clearly increased over ICU stay for $\mathrm{t}>0.6$. Higher cTIB thresholds resulted in larger increases to OR over time and were particularly significant for the 4.0-7.0 $\mathrm{mmol} / \mathrm{L}$ glycemic band.

Conclusion: Increased cTIB was associated with higher OL. These results suggest that effective GC positively influences patient outcome, regardless of how the glycemic regulation is achieved. $\mathrm{BG}<7.0 \mathrm{mmol} / \mathrm{L}$ is associated with a measurable increase in the odds of survival, if hypoglycemia is avoided.
\end{abstract}

Keywords: glycemia, critically ill patients, intensive care, mortality, glycemic control, critical care, patient outcome 


\section{Introduction}

High and variable blood glucose (BG) levels have been associated with increased mortality in critically ill patients ${ }^{1-5}$. Glycemic control (GC) has significantly reduced mortality ${ }^{6-8}$, but many studies have failed to reproduce these results ${ }^{9-12}$. These contradictory results have engendered skepticism towards GC in the critically ill patients ${ }^{13-16}$.

There are three main difficulties with GC. First, a high risk of hypoglycemia ${ }^{4,17-19}$, which is independently associated with poor outcome ${ }^{4,19}$, where only one study reduced hypoglycemia during $\mathrm{GC}^{8}$. Second, all GC protocols specify a limit or target band with the aim of reducing persistent hyperglycemia, to best ensure improved patient outcome. However, there is currently no expert consensus about a best GC target band ${ }^{13,20,21}$. Third, metrics of performance for glycemic control can typically only be evaluated at the end of the patient stay in the intensive care unit (ICU) ${ }^{22}$. However, it is important that clinicians are able to assess GC performance with respect to potential outcome at any time during stay to inform clinical decision-making.

The goal of this research is to demonstrate that well-regulated glycemia is beneficial to patient outcome, regardless of how it is achieved. Key to this demonstration is a metric that continuously and adequately captures the concept of 'well regulated.' We strongly agree with MacKenzie ${ }^{23}$ that both glycemic level and variability are essential components of wellregulated glycemia. Cumulative time in band (cTIB) is a simple metric that fulfills these criteria and enables us to investigate the impact of glycemia on patient outcome in terms of total exposure to pre-defined glycemic bands. Thus, we reanalyze data from two different glycemic control trials and use an odds ratio based on cTIB and mortality to show that certain glycemic bands are better than others. 


\section{Subjects and Methods}

\subsection{Patients}

This study used glycemic data from 1701 patients from two, independent studies:

1. SPRINT: Prospective SPRINT and retrospective pre-SPRINT cohorts were included in this study. These patients were admitted to Christchurch Hospital ICU between January 2003 and May $2007(\mathrm{~N}=784)^{8,24}$.

2. Glucontrol: A randomized multi-center study, admitted to ICUs from 3 November 2004 to 30 May $2006(\mathrm{~N}=917)^{9}$.

These two datasets represent very different ICU cohorts with conflicting results in GC trials. SPRINT reduced organ failure, mortality and hypoglycemia compared to the retrospective cohort ${ }^{8,24}$. The Glucontrol trial showed no benefit from GC to a low target compared to a higher target and, as is often the case, reported increased hypoglycemia for the low target cohort ${ }^{9}$. Local protocols had measurment frequency within the 1-4 hourly range for all periods where patients were glycaemically controlled. Patient data are summarized in Table 1 and the number of patients remaining in the ICU at each day are shown in Figure 1.

Table 1. Patient data shown as median [interquartile range] where appropriate.

\begin{tabular}{|lccc|}
\hline & $\begin{array}{c}\text { SPRINT } \\
\text { study }\end{array}$ & $\begin{array}{c}\text { Glucontrol } \\
\text { study }\end{array}$ & All \\
\hline Number of patients & 784 & 917 & 1701 \\
Percentage of males & 61.2 & 62.9 & 62.1 \\
Age of patients & $65.0[52.0-74.0]$ & $65.2[51.5-74.1]$ & $65.0[51.6-74.0]$ \\
APACHE 2 score & $18.0[15.0-24.0]$ & $15.0[11.0-21.0]$ & $17.0[13.0-23.0]$ \\
Cohort BG (mmol/L) & $6.2[5.3-7.4]$ & $6.9[5.8-8.4]$ & $6.6[5.6-8.1]$ \\
Per-patient median BG $(\mathrm{mmol} / \mathrm{l})$ & $6.3[5.6-7.5]$ & $6.9[6.1-8.2]$ & $6.6[5.8-7.9]$ \\
$\%$ BG in 4.0-7.0 mmol/L & 66.8 & 50.0 & 56.6 \\
$\begin{array}{l}\text { Number of patients with BG }<2.2 \\
\text { mmol/L }\end{array}$ & 36 & 54 & 90 \\
\hline
\end{tabular}




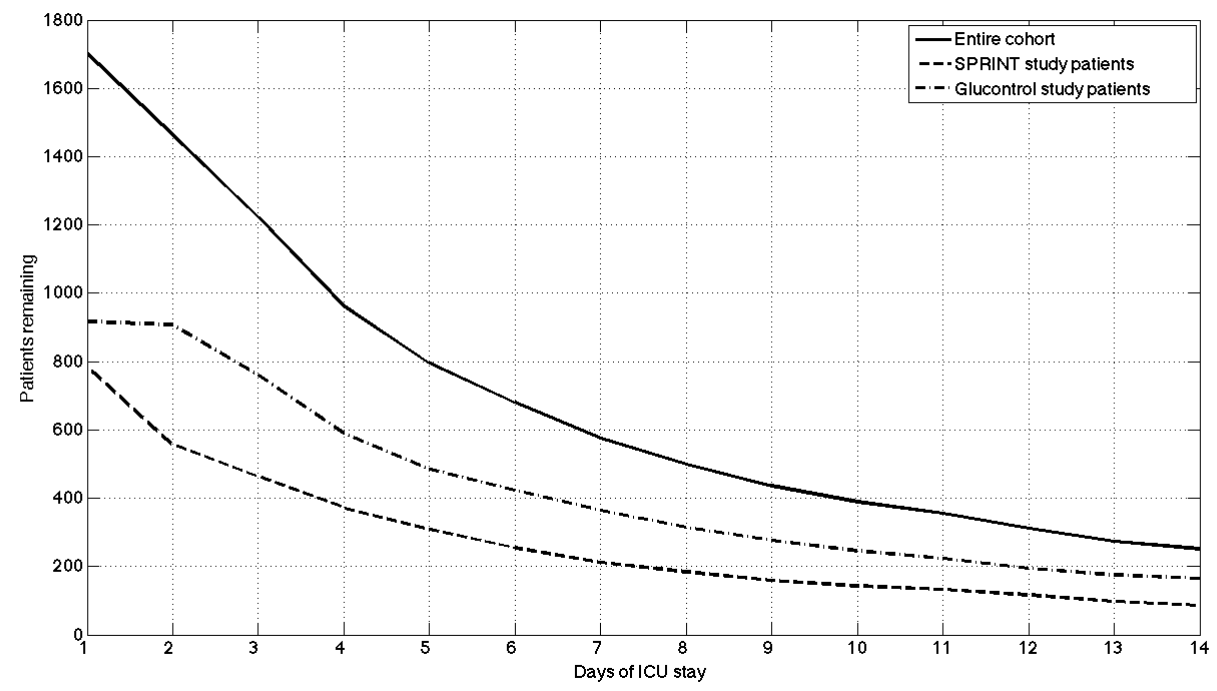

Figure 1. Patients remaining in the ICU at each day.

\subsection{Analysis}

Glycemic outcome and performance were measured using cumulative Time In Band (cTIB), calculated for each patient for each day of stay. cTIB is the time spent within the pre-defined glycemic band as a proportion of the total time up to and including the day under consideration ${ }^{24}$. Further, each patient day was classified into a category based on whether their cTIB value exceeded a pre-defined threshold, $t$, permitting a simple analysis of cohort behavior. Thus, for a given threshold, $t$, cTIB accounts simultaneously for both BG level and variability, where variability within the band is tolerated and more time (higher $t$ ) within a band of defined width limits overall variability.

To enable comparison, cTIB must be calculated from data with a constant measurement frequency. Clinical measurements from this retrospective data were not necessarily hourly, thus interpolated data were used in the calculation of cTIB when required. Across the entire cohort, the average duration between measurements was 2.5 hours. The analyses were performed for the first 14 days of glycemic monitoring, which typically commenced shortly after admission to the ICU. After 14 days, less that $15 \%$ of patients remained in the ICU.

For a specific day $d$, cTIB is calculated using Equation (1), where $\left(B G_{\text {day }(i)} \in\right.$ band $)$ are the hourly interpolated BG levels included in the defined glycaemic band.

$$
\operatorname{cTIB}(d)=\frac{\sum_{i=1}^{d}\left(B G_{\operatorname{day}(i)} \in \text { band }\right)}{\sum_{i=1}^{d} 24 * i}
$$

In this study, cTIB was calculated for the 4.0-7.0 mmol/L, 5.0-8.0 mmol/L and 4.0-8.0 mmol/L glycemic bands. These bands represent two different intermediate glycemic levels with similar 
tolerated variability $(4.0-7.0 \mathrm{mmol} / \mathrm{L}$ and $5.0-8.0 \mathrm{mmol} / \mathrm{L})$, and a wider band allowing more variability $(4.0-8.0 \mathrm{mmol} / \mathrm{L})$. These specific ranges were considered as they could reasonably be used as target bands for GC given current thinking ${ }^{2,25-27}$. Threshold values of $t=0.5,0.6$, 0.7 and 0.8 were considered, where a higher threshold value indicates less tolerance of dysglycemia. These thresholds were used as they represent achievable levels of quality control, and the value $t=0.5$ has been used previously to separate quality of control in association with clinical outcome ${ }^{24}$.

For each day during the first 14 days of ICU stay, patients were classified by cTIB, threshold and outcome hospital mortality, yielding a 2x2 contingency matrix for each day (2). Crucially, this classification was performed independent of the intention-to-treat groups and thus enables the analysis of the association between glycemic level and mortality, regardless of whether the glycemic control was achieved by protocol, natural regulation or a combination.

$$
\begin{array}{cc}
\mathrm{L} & \mathrm{D} \\
\operatorname{cTIB} \geq t \\
\operatorname{cTIB}<t
\end{array}\left[\begin{array}{ll}
N_{1} & N_{2} \\
N_{3} & N_{4}
\end{array}\right]
$$

The odds of living (OL) given cTIB $\geq t$ are defined as $N_{1} / N_{2}$ and similarly for cTIB $<t$. Where $N_{x}$ represents the number of patients that lived $(L)$ or died $(D)$ for each cTIB state. The odds ratio (OR), defined as the ratio of $\mathrm{OL}$ given $\mathrm{cTIB} \geq t$ to $\mathrm{OL}$ given cTIB $<t$ :

$$
\mathrm{OR}=\frac{N_{1} N_{4}}{N_{2} N_{3}}
$$

Equation (4) describes the $95 \%$ confidence interval about OR ${ }^{28}$ :

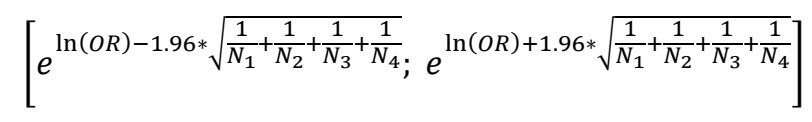

For each day of ICU stay, OL and OR, with 95\% confidence interval (CI), were calculated for the cohort. The association between glycemic performance (defined by the cTIB metric) and mortality outcome was tested using the Chi-squared test with the contingency matrix (2). 


\section{Results}

Figure 2 shows the odds of living, by day for the combined cohort for each band and threshold $(t)$. The asterisks indicate a statistically significant $(\mathrm{p}<0.05$, chi-squared test) association between cTIB $\geq t$ and mortality. Figure 3 similarly presents the evolution of OR over time with associated confidence intervals.

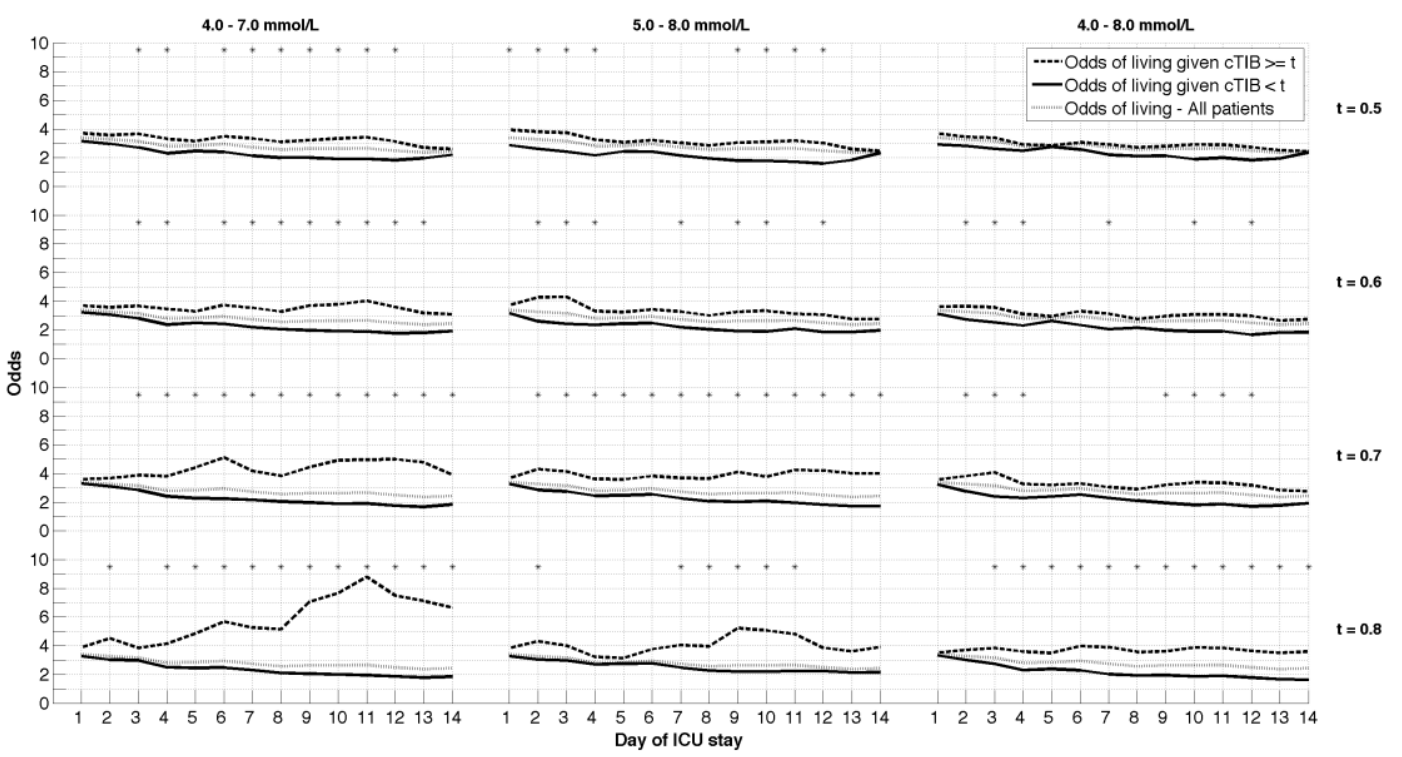

Figure 2. Cohort odds of living for each glycemic band and threshold value during ICU stay.

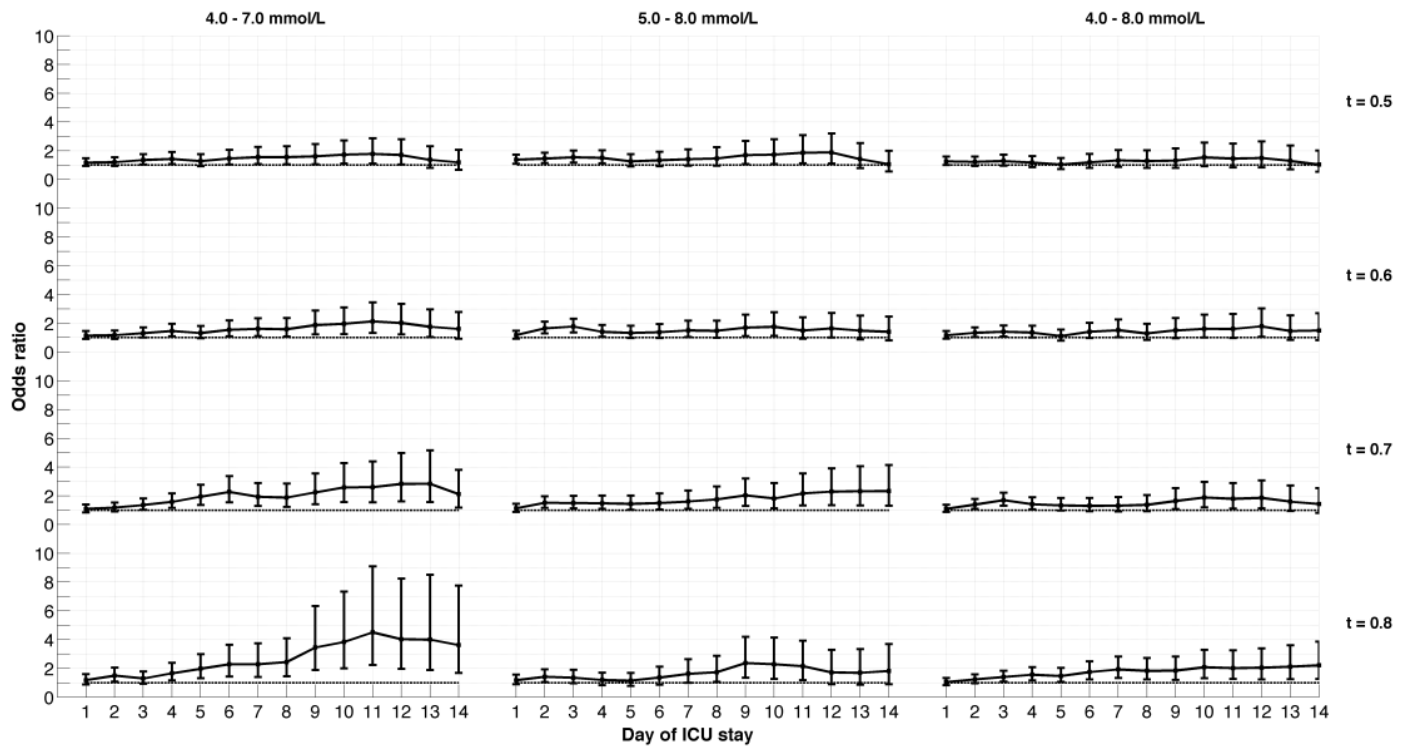

Figure 3. Cohort odds ratio for each band and threshold during ICU stay.

When considering separately results for patients who experienced hypoglycemia (at least on $\mathrm{BG}<2.2 \mathrm{mmol} / \mathrm{L}$ ) and patients who did not (Supplemental file), results showed that for patients experiencing severe hypoglycemia the odds ratio is not different than 1.0. Those patients who 
did not experience severe hypoglycemia had very similar odds ratio values compared to the entire cohort, with differences being clinically and numerically very small to negligible as a result of the relatively lower numbers of severe hypoglycemic events over the large cohort. Thus, compared to the entire cohort as a whole, severe hypoglycemia significantly reduces odds ratios, but avoiding it did not significantly improve them. The Supplemental file repeats Figure 3 for comparison for both the severe hypoglycemia cohort and the cohort who did not experience hypoglycemia 


\section{Discussion}

Two key factors influence GC in the ICU. First, is the physiological question: Does adequately regulated BG benefit patients? The second, and arguably more difficult, factor is the actual implementation of successful, accurate GC in a busy ICU environment. Van den Berghe et al.

${ }^{7,27}$ separated these factors by using a specialist nursing team and focused on the physiological question, demonstrating the benefit of GC on patient outcome. A number of studies added weight to this finding by pinning down the pathophysiological mechanisms and pathways behind glucose toxicity ${ }^{29-33}$. This study is unique in that it analyses the combined results from two studies, in normal clinical settings, based on glycemic level, rather than the treatment group. It thus effectively separates physiology from implementation.

It is immediately clear from Figure 2 that $\mathrm{OL}$ given $\mathrm{cTIB} \geq t$ is higher than $\mathrm{OL}$ given $\mathrm{cTIB}<t$ for all values of $t$, every day, for all three glycemic bands studied. Furthermore, Figure 3 shows that the difference between the odds clearly increased over ICU stay for $\mathrm{t}=0.7$ and 0.8 . In each case, the odds ratio tended to increase over ICU stay until Day 11. Higher cTIB thresholds resulted in larger increases to OR over time and were particularly significant for the 4.0$7.0 \mathrm{mmol} / \mathrm{L}$ glycemic band.

In particular, in this study, the three bands and various thresholds, $t$, used test different acceptable levels and variability of glycemia. The comparison of the 4.0-7.0 and 5.0-8.0 $\mathrm{mmol} / \mathrm{L}$ bands for a given value of $\mathrm{t}$ effectively compare the average glycemic level achieved. Comparison to the wider $4.0-8.0 \mathrm{mmol} / \mathrm{L}$ band assesses the impact of increased potential variability allowed by a wider band width. In Figure 3, the results show advantage for lesser variability and a lower glycemic level, where increasing strictness with rising threshold, t, yields better results across all bands.

This study's results clearly demonstrate a strong association between accurate glycemic control and mortality, regardless of how the glycemic regulation came about. Regulated glycemia was considered equally good whether it was due to a tight GC protocol, endogenous regulation, or a combination. In particular, more time spent within the 4.0-7.0 $\mathrm{mmol} / \mathrm{L}$ glycemic band was associated with higher odds of survival compared to the higher and wider bands. Moreover, patients who did not experience severe hypoglycemia during their ICU stay had slightly higher odds ratio values than the entire cohort. Those had at least one $\mathrm{BG}<2.2 \mathrm{mmol} / \mathrm{L}$ had odds ratios indistinguishable from 1.0, although this cohort was relatively quite small. This finding matches results in the literature suggesting the negative effects of severe hypoglycemia. In concert with Figure 3, they suggest that safe, effective control in a $4.0-7.0 \mathrm{mmol} / \mathrm{L}$ range, as defined here using cTIB, is associated with a measurable increase in the odds of survival, if hypoglycemia is avoided. 
A possible reason why randomized controlled GC trials yielded conflicting results is that they targeted glycemic level with no means to directly manage variability. These results thus suggest that protocols that directly minimize variability within a specific target band (i.e. level and variability) should be prospectively tested to ascertain whether there is a causal relationship with outcome. Hence, this result is not inconsistent with the latest results of the NICE-SUGAR study ${ }^{10}$, and other recent reports. Future studies could also examine the ability to achieve given thresholds for specific sub-cohorts based on diagnosis, organ failure, or other severity score, over time.

An important aspect of this study was the use of the cumulative time in band metric. This metric captures both the level and variability of glycemia, as well as relative exposure to dysglycemia. The cTIB metric was shown to be strongly associated with patient outcome, particularly after 3 days of ICU stay, as indicated by the lower limits of the $95 \%$ confidence intervals of Figure 3. Chase et al. and Van den Berghe et al. ${ }^{8,27}$ also reported reduced mortality after 3 or more days of glycemic control. Therefore, cTIB provides a simple, yet useful metric for clinicians and investigators to evaluate the evolution of glycemic control in patients in real-time.

The choices of glycemic ranges investigated in this study were not arbitrary. Several studies have shown that targeting $\mathrm{BG} \leq 8.1 \mathrm{mmol} / \mathrm{l}$ is beneficial ${ }^{2,25}$. However, fear of hypoglycemia has driven suggested target ranges higher ${ }^{21}$. Thus, the ranges investigated in this study were intermediate and achievable, provided the metabolic variability leading to hypo- and hyperglycemia can be managed ${ }^{34}$.

Clinically, in consideration of the pathophysiological basis of increasing cellular dysfunction with dysglycemia, this cTIB metric captures glycemic variability and glycemic level in combination with the length of exposure to these effects. This metric can readily be targeted by control protocols and evaluated regularly (daily or more frequently) in real-time at the patient's bedside.

It is also interesting to note that by Day 11 or 12 , the odds ratio starts deceasing in some cases in Figure 3. However, by this point only $20 \%$ of the patients are remaining (Figure 1) who are likely very critically ill. Thus, these results may be explained by the fact that by that time only the very ill, longer staying patients are left.

The limitations of this study should be acknowledged. First, BG measurement frequency varied between patients and centers. To use the cTIB metric, the data needed to have a constant and consistent frequency, thus BG measurements were interpolated to provide one value per hour. As the cTIB metric is a cumulative method for quantifying glycemic behavior over time, we feel that interpolation is justified, as it captures the average trend of the BG between the measurements. 
Second, this study was performed on retrospective data, thus we can only note the association between well-regulated glycemia and outcome mortality and cannot prove causation. However, others ${ }^{29-33}$ have determined pathophysiological pathways between hyperglycemia, glycemic variability and negative outcomes. So, although well-regulated glycemia may be a symptom of more healthy patients, rather than a cause, we feel that the evidence from numerous pathophysiological and clinical studies suggests that the association seen in this study results from an underlying causative pathway, which should be further investigated prospectively.

Third, the analysis groups all cohorts and patients together. It thus considers the glycemic outcomes of a large, heterogeneous cohort made of different types of patients who were treated differently in terms of insulin. The glycemic outcomes were thus variable, and due to the different quality of control protocols used, did not necessarily match expectations, where less ill cohorts by APACHE score had sometimes higher glycemic levels and variability. As a result, the study cannot clearly define or delineate for which sub-cohort, such as surgical patients in [7] or the medical ICU patients of [8], the results are better or worse.

However, the main objective of this study was to demonstrate an association between level and variability of glycemic control achieved, regardless of how that outcome was achieved or by what protocol, and patient outcome. It was not intended to link the ability to achieve a glycemic outcome and the level of illness which remains to be defined, although the SPRINT controlled group of [8] did consistently achieve control over such a broad cohort as seen in [24]. Hence, as a final limitation, the results presented thus offer a stepping stone for greater delineation and refinement with larger cohorts in additional studies. 


\section{Conclusions}

Increased cumulative time in an intermediate glycemic band was associated with higher odds of living. These results suggest that effective GC positively influences patient outcome, regardless of how the glycemic regulation is achieved. There were significant differences in the glycemic bands studied with a 4.0-7.0 mmol/L band showing improved results over a similar width band between 5.0-8.0 mmol/L, indicating that $\mathrm{BG}$ lower than $7.0 \mathrm{mmol} / \mathrm{L}$ is associated with a measurable increase in the odds of survival, if hypoglycemia is avoided. Hence, these results demonstrate an association between the level and tightness of control, regardless of how it is achieved, and outcome, while offering a foundation to further studies to clarify the link between severity of illness and ability to achieve a glycemic level and variability.

The cTIB metric can be readily calculated in real-time and used to assess GC in progress, as well as providing a useful, simple target for GC studies. The single metric encapsulates the need to achieve control of both level and variability to minimize cellular dysfunction, as well as linking the level of achievement to patient outcome over each day of stay. 


\section{Abbreviations}

BG: blood glucose

CI: confidence interval

cTIB: cumulative time in band

GC: glycemic control

ICU: intensive care unit

OL: odds of living

OR: odds ratio 


\section{Competing interests}

The authors declare that they have no competing interests. 


\section{Authors' contribution}

All authors were involved in the analysis and interpretation of data. The manuscript was originally drafted by SP, JGC and JCP, but all authors made contributions through the entire process, including reading and final approval of this manuscript. 


\section{Authors' information}

1. MSc, Cardiovascular Research Centre, Institut de Physique, Université de Liege, Institut de Physics, Allée du 6 Août, 17 (Bât B5), B4000 Liege, Liege, Belgium.

2. $\mathrm{PhD}$, Department of Mechanical Engineering, Centre for Bio-Engineering, University of Canterbury, Christchurch, Private Bag 4800, 8054, New Zealand.

3. PhD, Department of Intensive Care, Erasme University Hospital, 808 route de Lennik, B1070 Brussels, Belgium.

4. MB, ChB, Department of Intensive Care, Christchurch Hospital, Christchurch, 8054, New Zealand

5. $\mathrm{PhD}$, Department of Mechanical Engineering, Centre for Bio-Engineering, University of Canterbury, Christchurch, Private Bag 4800, 8054, New Zealand.

6. $\mathrm{PhD}$, Cardiovascular Research Centre, Institut de Physique, Université de Liege, Institut de Physics, Allée du 6 Août, 17 (Bât B5), B4000 Liege, Liege, Belgium. 


\section{References}

1. Krinsley JS: Glycemic variability: a strong independent predictor of mortality in critically ill patients. Crit Care Med 2008, 36(11):3008-3013.

2. Krinsley JS: Association between hyperglycemia and increased hospital mortality in a heterogeneous population of critically ill patients. Mayo Clinic proceedings Mayo Clinic 2003, 78(12):1471-1478.

3. Egi M, Bellomo R, Stachowski E, French CJ, Hart G: Variability of blood glucose concentration and short-term mortality in critically ill patients. Anesthesiology 2006, 105(2):244-252.

4. Bagshaw SM, Bellomo R, Jacka MJ, Egi M, Hart GK, George C: The impact of early hypoglycemia and blood glucose variability on outcome in critical illness. Critical care 2009, 13(3):R91.

5. McCowen KC, Malhotra A, Bistrian BR: Stress-induced hyperglycemia. Crit Care Clin 2001, 17(1):107-124.

6. Krinsley JS: Effect of an intensive glucose management protocol on the mortality of critically ill adult patients. Mayo Clinic proceedings Mayo Clinic 2004, 79(8):992-1000.

7. van den Berghe G, Wouters P, Weekers F, Verwaest C, Bruyninckx F, Schetz M et al: Intensive insulin therapy in critically ill patients. The New England journal of medicine 2001, 345(19):1359-1367.

8. Chase JG, Shaw G, Le Compte A, Lonergan T, Willacy M, Wong XW et al: Implementation and evaluation of the SPRINT protocol for tight glycaemic control in critically ill patients: a clinical practice change. Critical care 2008, 12(2):R49.

9. Preiser JC, Devos P, Ruiz-Santana S, Melot C, Annane D, Groeneveld J et al: A prospective randomised multi-centre controlled trial on tight glucose control by intensive insulin therapy in adult intensive care units: the Glucontrol study. Intensive care medicine 2009, 35(10):1738 - 1748.

10. Finfer S, Chittock DR, Su SY, Blair D, Foster D, Dhingra V et al: Intensive versus conventional glucose control in critically ill patients. N Engl J Med 2009, 360(13):1283-1297.

11. Brunkhorst FM, Engel C, Bloos F, Meier-Hellmann A, Ragaller M, Weiler N et al: Intensive insulin therapy and pentastarch resuscitation in severe sepsis. N Engl J Med 2008, 358(2):125-139. 
12. Griesdale DE, de Souza RJ, van Dam RM, Heyland DK, Cook DJ, Malhotra A et al: Intensive insulin therapy and mortality among critically ill patients: a meta-analysis including NICE-SUGAR study data. Cmaj 2009.

13. Anger KE, Szumita PM: Barriers to glucose control in the intensive care unit. Pharmacotherapy 2006, 26(2):214-228.

14. Egi M, Finfer S, Bellomo R: Glycemic control in the ICU. Chest 2011, 140(1):212220.

15. Inzucchi SE, Siegel MD: Glucose control in the ICU--how tight is too tight? The New England journal of medicine 2009, 360(13):1346-1349.

16. Lomangino K: Glycemic Control in the ICU: Enthusiasm Fading for "Tight" Targets. Clinical Nutrition Insights 2007, 33(10):10-13.

17. Falciglia M, Freyberg RW, Almenoff PL, D'Alessio DA, Render ML: Hyperglycemiarelated mortality in critically ill patients varies with admission diagnosis. Crit Care Med 2009, 37(12):3001-3009.

18. Krinsley JS, Keegan MT: Hypoglycemia in the critically ill: how low is too low? Mayo Clinic proceedings Mayo Clinic 2010, 85(3):215-216.

19. Investigators N-SS, Finfer S, Liu B, Chittock DR, Norton R, Myburgh JA et al: Hypoglycemia and risk of death in critically ill patients. N Engl J Med 2012, 367(12):11081118.

20. Ichai C, Preiser JC, Societe Francaise dA-R, Societe de Reanimation de langue F, Experts g: International recommendations for glucose control in adult non diabetic critically ill patients. Critical care 2010, 14(5):R166.

21. Moghissi ES, Korytkowski MT, DiNardo M, Einhorn D, Hellman R, Hirsch IB et al: American Association of Clinical Endocrinologists and American Diabetes Association consensus statement on inpatient glycemic control. Diabetes Care 2009, 32(6):1119-1131.

22. Eslami S, de Keizer NF, de Jonge E, Schultz MJ, Abu-Hanna A: A systematic review on quality indicators for tight glycaemic control in critically ill patients: need for an unambiguous indicator reference subset. Critical care 2008, 12(6):R139.

23. Mackenzie IM, Whitehouse T, Nightingale PG: The metrics of glycaemic control in critical care. Intensive Care Med 2011, 37(3):435-443.

24. Chase JG, Pretty CG, Pfeifer L, Shaw GM, Preiser JC, Le Compte AJ et al: Organ failure and tight glycemic control in the SPRINT study. Crit Care 2010, 14(4):R154. 
25. Al-Tarifi A, Abou-Shala N, Tamim HM, Rishu AH, Arabi YM: What is the optimal blood glucose target in critically ill patients? A nested cohort study. Annals of thoracic medicine 2011, 6(4):207-211.

26. Evans A, Shaw GM, Le Compte A, Tan CS, Ward L, Steel J et al: Pilot proof of concept clinical trials of Stochastic Targeted (STAR) glycemic control. Ann Intensive Care 2011, 1:38. 27. Van den Berghe G, Wilmer A, Hermans G, Meersseman W, Wouters PJ, Milants I et al: Intensive Insulin Therapy in the Medical ICU. N Engl J Med 2006, 354(5):449-461.

28. Motulsky HJ: Biostatistique: Une approche intuitive: de boeck; 2002.

29. Brownlee M: Biochemistry and molecular cell biology of diabetic complications. Nature 2001, 414(6865):813-820.

30. Langouche L, Vanhorebeek I, Vlasselaers D, Vander Perre S, Wouters PJ, Skogstrand $\mathrm{K}$ et al: Intensive insulin therapy protects the endothelium of critically ill patients. $J$ Clin Invest 2005, 115(8):2277-2286.

31. Van den Berghe G: How does blood glucose control with insulin save lives in intensive care? J Clin Invest 2004, 114(9):1187-1195.

32. Weekers F, Giulietti AP, Michalaki M, Coopmans W, Van Herck E, Mathieu C et al: Metabolic, endocrine, and immune effects of stress hyperglycemia in a rabbit model of prolonged critical illness. Endocrinology 2003, 144(12):5329-5338.

33. Siegelaar SE, Holleman F, Hoekstra JB, DeVries JH: Glucose variability; does it matter? Endocr Rev 2010, 31(2):171-182.

34. Chase JG, Le Compte AJ, Suhaimi F, Shaw GM, Lynn A, Lin J et al: Tight glycemic control in critical care--the leading role of insulin sensitivity and patient variability: a review and model-based analysis. Comput Methods Programs Biomed 2011, 102(2):156-171. 


\section{Figure captions}

Figure 1. Patients remaining in the ICU at each day.

Figure 2. Cohort odds of living for each glycemic band and threshold value during ICU stay.

Figure 3. Cohort odds ratio for each band and threshold during ICU stay. 


\section{Table captions}

Table 1. Patient data shown as median [interquartile range] where appropriate. 